\title{
Solution of axisymmetric crack problems using distributed dislocation ring dipoles
}

\author{
A M Korsunsky and D A Hills* \\ Department of Engineering Science, University of Oxford, UK
}

\begin{abstract}
A solution procedure for the calculation of crack tip stress intensity factors arising at the edges of an arbitrarily shaped crack lying on a surface of revolution is described. The cracks are subject to an arbitrary axisymmetric stress field, devoid of torsion, and may be present in either an infinite space or two elastically dissimilar bonded half-spaces, of which one may, as a special case, have vanishing elastic constants. The technique employed is a one-dimensional integral equation approach, in which the kernel is formed from rings of dislocation pairs, arranged to form ring 'dipoles'. The equation is hypersingular but may readily be inverted using powerful numerical quadratures, providing a computationally efficient solution. Examples of the use of the technique are then described.
\end{abstract}

Keywords: crack problems, singular boundary integral equation, stress intensity factor

\section{NOTATION}

$\begin{array}{ll}a & \text { ring radius } \\ b_{i} & \text { Burgers vector component in direction } i \\ b_{i j} & \text { dipole intensity } \\ B_{i} & \text { dislocation density } \\ \mathbf{E} & \text { complete elliptic integral, second kind } \\ k & \text { elliptic modulus } \\ K_{j}^{i}, L_{j}^{i}, D_{k l}^{i j} & \text { influence functions } \\ r, \theta, z & \text { cylindrical polar coordinates } \\ x, \xi, t, s & \text { crack line coordinates } \\ \delta_{i j} & \text { Kronecker delta } \\ \kappa & \text { Kolosov's constant }=3-4 v \\ \mu & \text { shear modulus } \\ v & \text { Poisson's ratio } \\ \sigma_{i j} & \text { stress components } \\ \rho & \text { normalized coordinates }=r / a \text { and }\left(z-z^{1}\right) / a \\ & \text { respectively }\end{array}$

\section{INTRODUCTION}

The idea of using the Bueckner [1] superposition theorem to solve for crack tip stress intensity factors is well established. First, the stresses arising along the line of the crack, but in its absence are found, and then equal and opposite equilibrating tractions are installed along the faces

\footnotetext{
The MS was received on 16 August 1999 and was accepted after revision for publication on 25 January 2000.

* Corresponding author: Department of Engineering Science, University of Oxford, Parks Road, Oxford OX1 3PJ, UK.

of the crack, to render them stress free. The contributions that these stresses make to the crack tip stress intensity are then deduced and, from the Bueckner theorem, these must be equal to the stress intensity developed by the original loading. The entities used to induce the equilibrating stress state are invariably some form of strain nucleus, whose exact form depends on whether the problem is two or three dimensional. The intention in the present paper is to present a new form of the procedure, appropriate to axisymmetric problems devoid of torsion. Although only restricted geometries may be tackled, such as cracks present in an infinite space or a half-space, the method is quite general, insofar as any nominal stress field may be handled; the technique is therefore particularly appropriate where the crack exists in a steep stress gradient, such as adjacent to a contact or elastic interface, and where the presence of far boundaries has only a very weak influence on the solution, e.g. when the crack is quite short by comparison with other characteristic dimensions.

It is illuminating to begin to describe the method by comparing it with the solution of plane crack problems by the installation of dislocations, and the solution of flat, arbitrarily shaped cracks by the eigenstrain procedure, as shown in Fig. 1. In the case of plane cracks (Fig. 1a), the solution used to provide the Green's function for the equilibrating stress field is the straight edge dislocation. There are many papers in the literature which apply this approach, some dating back to the early 1970s (see, for example, references [2] and [3]). A simple exposition of the procedure was given by Nowell and Hills [4], which might be consulted as an introduction. The first step in the solution procedure is to establish the stress state induced by a single dislocation, which takes the form 
(a)

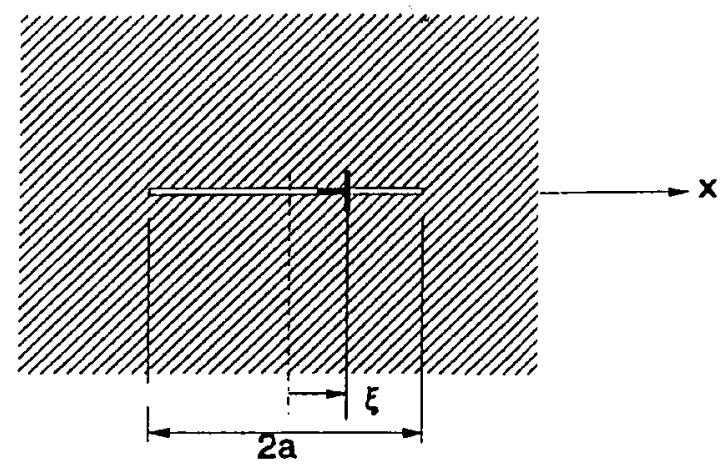

(b)

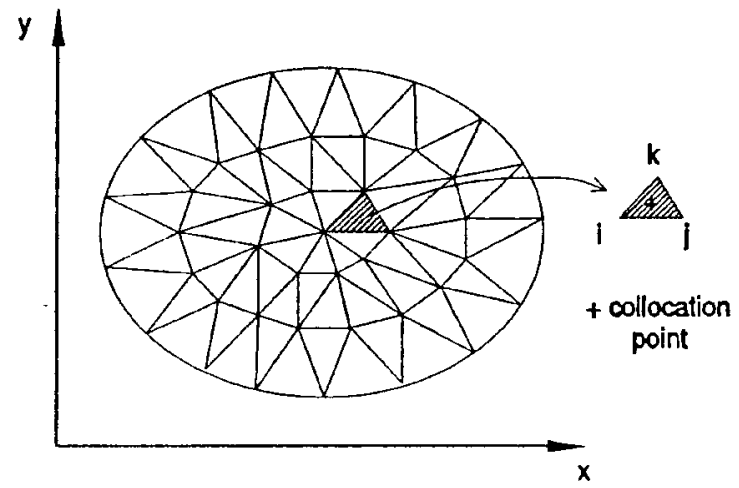

(c)

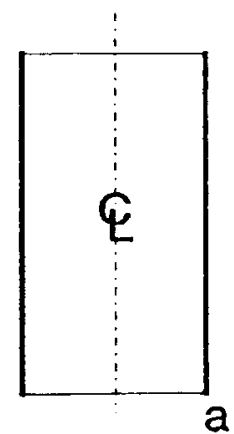

(d)

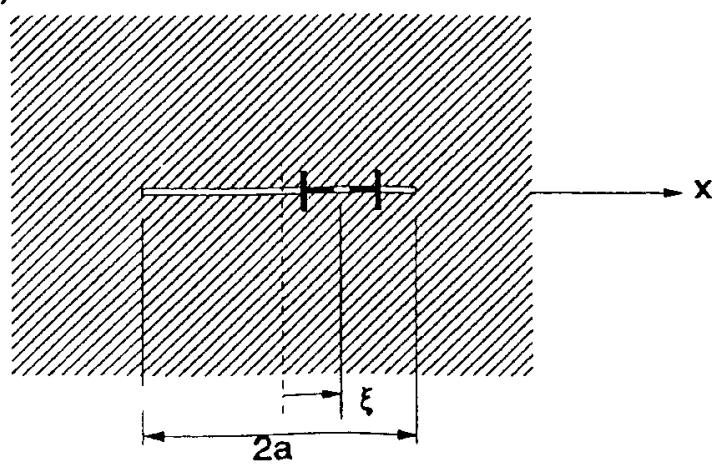

Fig. 1 Possible kinds of strain nucleus: (a) the edge dislocation for plane problems; (b) the eigenstrain-type procedure using infinitesimal dislocation loops, for general threedimensional problems; (c) the axisymmetric ring dipole; (d) the dislocation dipole for plane problems

$$
\begin{aligned}
\sigma_{x y}(x)=\frac{\mu}{\pi(1+\kappa)}\{ & {\left[\frac{1}{x-\xi}+K_{x}^{x}(x, \xi)\right] b_{x}(\xi) } \\
& \left.+K_{x}^{y}(x, \xi) b_{y}(\xi)\right\} \\
\sigma_{y y}(x)=\frac{\mu}{\pi(1+\kappa)}\{ & K_{y}^{x}(x, \xi) b_{x}(\xi) \\
+ & {\left.\left[\frac{1}{x-\xi}+K_{y}^{y}(x, \xi)\right] b_{y}(\xi)\right\} }
\end{aligned}
$$

Here, $\mu$ is the modulus of rigidity, $\kappa$ is Kolosov's constant [ $=(3-4) v$, where $v$ is Poisson's ratio, in plane strain], and the four functions $K_{j}^{i}$ represent the influence of the far boundaries of the problem but are regular. The requirement that the crack faces be traction free is then expressed by the equations

$$
\begin{aligned}
& \begin{aligned}
0=\tilde{\sigma}_{x y}(x)+\frac{\mu}{\pi(1+\kappa)}\{ & \left\{\int_{\text {crack }}\left[\frac{1}{x-\xi}+K_{x}^{x}(x, \xi)\right] B_{x}(\xi)\right. \\
& \left.+K_{x}^{y}(x, \xi) B_{y}(\xi)\right\} \mathrm{d} \xi \\
0=\tilde{\sigma}_{y y}(x)+\frac{\mu}{\pi(1+\kappa)}\{ & \left\{\int_{\text {crack }} K_{y}^{x}(x, \xi) B_{x}(\xi)\right. \\
+ & {\left.\left[\frac{1}{x-\xi}+K_{y}^{y}(x, \xi)\right] B_{y}(\xi)\right\} \mathrm{d} \xi }
\end{aligned}
\end{aligned}
$$

where $\tilde{\sigma}_{i y}(x)$ are the tractions arising in the crack's absence, due to the far field loading, and the functions $B_{i}(\xi)$ represent dislocation densities, defined by

$$
B_{i}(\xi)=\frac{\partial b_{i}}{\partial \xi}
$$

form the primary unknowns in the problem. In certain cases the 'cross terms' $K_{y}^{x}$ and $K_{x}^{y}$ vanish, but in general two coupled integral equations, with Cauchy kernels, must be solved. Powerful numerical quadratures are available to deal with this [3], and relatively few simultaneous algebraic equations may be used to obtain a satisfactory solution. Crack tip stress intensity factors may be found from the values of the dislocation densities at the end points [4-6]. An alternative basis for solving plane crack problems was proposed by Crouch [7], who derived analytical expressions for stresses and displacements around a straight segment carrying prescribed uniform displacement discontinuity. A constant-gap boundary element formulation results.

The more complex case of a three-dimensional crack is shown in Fig.1b. This can also be tackled using boundary element approaches [8]. An eigenstrain procedure may also be developed, which is an analogue of the scheme described above. The kernel of the equations is formed 
from a solution for the stress state induced by insertion of a thin strip of material (in the case of mode one loading), over an infinitesimal dislocation loop. In principle, it is possible to write down a two-dimensional integral equation over the crack faces, $S$, expressing the traction-free condition over the entire crack, which takes the form

$$
\begin{aligned}
0=\tilde{\sigma}_{i}(\boldsymbol{x})+\frac{\mu}{4 \pi(1-v)} \int_{S} & \left\{\frac { 1 } { r ^ { 3 } } \left[(1-2 v) \delta_{i j}+2 v \delta_{3 i} \delta_{3 j}\right.\right. \\
+ & \left.\left.+3 v \frac{r_{i} r_{j}}{r^{2}}\right]+K_{i j}(\boldsymbol{x}, \boldsymbol{y})\right\} b_{j}(\boldsymbol{y}) \mathrm{d} S
\end{aligned}
$$

where $\delta_{i j}$ is the Kronecker delta, $\boldsymbol{r}=\boldsymbol{x}-\boldsymbol{y}$ and again $K_{i j}(\boldsymbol{x}, \boldsymbol{y})$ represents the influence of the free boundaries. It may be noted that the integral is nominally divergent, the kernel being hypersingular. No general closed-form inversion procedures are available, so that numerical procedures must be applied $[9,10]$. The crack may be divided into a finite number of elements, within each of which infinitesimal dislocation loops are distributed according to some pre-determined function (the shape function). For example, for a piecewise constant discretization the entire element suffers a constant displacement, equivalent to the Burgers vector of the constituent dislocation loops. The tractionfree condition is then enforced in an average sense, over the faces of the crack. This procedure is efficient, compared with the finite element technique, for cracks in simple bodies, and it may, in principle, be employed for cracks which are not flat, such as the tubular form shown in Fig. 1c. However, there are clearly disadvantages in using eigenstrain elements pasted around the curved surface of the crack, as no advantage is being taken of the inherent symmetry of the problem. A new kernel is therefore required, which exploits symmetry. This kernel may be formed from rings in the form of self-annihilating pairs of ring dislocations, which we have called ring dipoles. Before introducing them for the axisymmetric problem, it is worth revisiting the plane problem of Fig. 1a, and noting that a different kernel which consists of two equal and opposite dislocations an infinitesimal distance apart (Fig. 1d) may be employed. This is the plane form of a dislocation dipole. The resulting integral equations are [5]

$$
\begin{aligned}
& 0=\tilde{\sigma}_{x y}(x)+\frac{\mu}{\pi(1+\kappa)}\left\{\int_{\text {crack }}\left[\frac{1}{(x-\xi)^{2}}+L_{x}^{x}(x, \xi)\right] b_{x}(\xi)\right. \\
& \left.+L_{x}^{y}(x, \xi) b_{y}(\xi)\right\} \mathrm{d} \xi \\
& 0=\tilde{\sigma}_{y y}(x)+\frac{\mu}{\pi(1+\kappa)}\left\{\int_{\text {crack }} L_{y}^{x}(x, \xi) b_{x}(\xi)+\left[\frac{1}{(x-\xi)^{2}}\right.\right. \\
& \left.\left.+L_{y}^{y}(x, \xi)\right] b_{y}(\xi)\right\} \mathrm{d} \xi
\end{aligned}
$$

The functions $L_{j}^{i}(x, \xi)$ represent the influence of any far boundaries and are regular functions. This implies that they contain no singularities, provided that the points $\xi$ and $x$ do not lie on a free surface simultaneously.

The dipole influence function may be developed by placing two equal and opposite dislocations an infinitesimal distance, $\mathrm{d} \xi$ apart. Hence it may be readily shown that the new kernels are simply the derivatives of those used in equations (1) and (2):

$$
L_{j}^{i}(x, \xi)=\frac{\partial K_{j}^{i}(x, \xi)}{\partial \xi}
$$

The quantities $b_{i}(\xi)$, forming the primary unknowns, now take on the role of dislocation dipole densities, and they therefore have the same dimensions as the Burgers vector. Quadratures for the numerical solution of equations such as equation (5), containing a hypersingular term of the second degree, have been developed by Kaya and Erdogan [11]. Solution of the crack plane problems addressed by this method shows a small advantage over the distributed dislocation approach, insofar as the truncated family of algebraic equations used to represent the integral may be reduced in size, for a given degree of accuracy required.

Now the axisymmetric formulation itself is considered.

\section{FORMULATION}

The general class of problems to be solved is shown in Fig. 2a. They are various kinds of cracks of revolution, which will be assumed to be subjected to a general remote set of forces, axisymmetric in nature and devoid of torsion. The first step in the solution of these problems is to determine kernel functions corresponding to the dislocation dipoles needed. It is most convenient to develop these in a cylindrical coordinate set, and the three quantities needed are shown in Fig. 2b. They are as follows:

1. The dipole $b_{z z}$ is formed from two prismatic dislocation loops, having a Burgers vector component $\pm b_{z}$, positioned a small radial distance, $\mathrm{d} \xi$ apart, on the same $z=$ constant plane. It may be noted that the constituent dislocations are of the conventional Volterra type, with a constant Burgers vector at all points around the crack front. Dipoles of this kind alone would suffice for solving for the stress intensitities arising at the edges of flat annular cracks undergoing opening mode loading only.

2. The dipole $b_{r r}$ is formed from two ring dislocations having a radial Burgers vector component $\pm b_{r}$, positioned a small axial distance $\mathrm{d} \xi$ apart, on the same $r=$ constant surface. This dipole is needed to study opening mode loading of cylindrical cracks.

3. The shear-type dipole $b_{r z}\left(\equiv b_{z r}\right)$ is formed in one of two ways: either from two dislocations of Burgers vector 


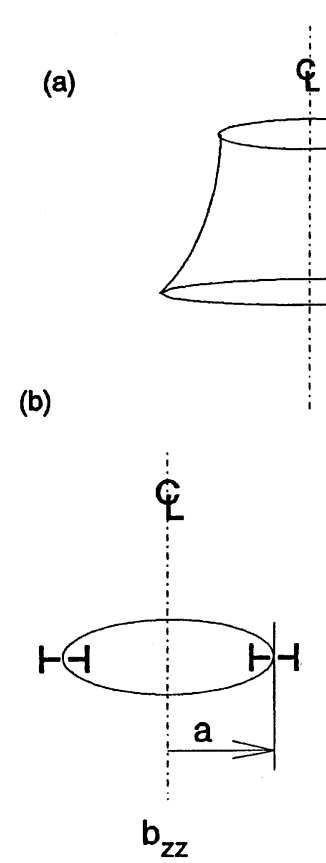

(i)

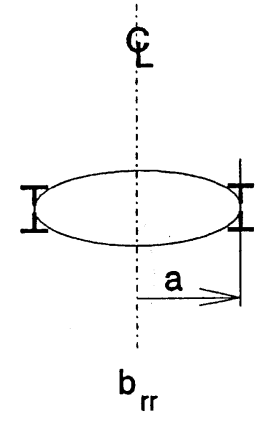

(ii)

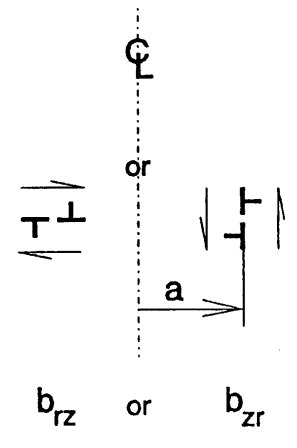

(iii)

Fig. 2 (a) General form of axisymmetric crack which might be solved by the procedure; (b) the three kinds of ring dipole needed

component $b_{r}$ positioned a small distance $\mathrm{d} \xi$ apart on a $z=$ constant surface, or from two dislocations of Burgers vector component $b_{z}$ positioned a small distance $d_{\xi}$ apart on an $r=$ constant surface.

The state of stress induced by each of these entities has been found for three configurations, namely an infinite space, a half-space or two elastically dissimilar bonded half-spaces. It is not the intention of the present paper to derive these expressions here, for which the paper by Korsunsky [12] should be consulted. It is merely noted that the state of stress induced at point $(r, z)$ by a dipole positioned at point $\left(a ; z^{\prime}\right)$, as depicted in Fig. 3, may be written in the form

$$
\begin{aligned}
\left\{\begin{array}{c}
\sigma_{z z}(r, z) \\
\sigma_{r r}(r, z) \\
\sigma_{r z}(r, z)
\end{array}\right\}= & \frac{2 \mu}{\pi(\kappa+1)}\left\{b_{z z}\left(a ; z^{\prime}\right)\left[\begin{array}{c}
D_{z z}^{z z} \\
D_{r r}^{z z} \\
D_{r z}^{z z}
\end{array}\right]\right. \\
& \left.+b_{r r}\left(a ; z^{\prime}\right)\left[\begin{array}{c}
D_{z z}^{r r} \\
D_{r r}^{r r} \\
D_{r z}^{r r}
\end{array}\right]+b_{r z}\left(a ; z^{\prime}\right)\left[\begin{array}{c}
D_{z z}^{r z} \\
D_{r r}^{r z} \\
D_{r z}^{r z}
\end{array}\right]\right\}
\end{aligned}
$$

The functions $D_{k l}^{i j}$ are recorded in Appendix 1, for the case of a dipole present in an infinite space. It will be noted that the functions quoted there allude to a further family of functions $J_{m n p}$ which are known as modified LipschitzHankel integrals. These contain combinations of Bessel functions, but with the arguments encountered they may be reduced to combinations of the complete elliptic integrals $\mathbf{K}$ and $\mathbf{E}$.

From work on the plane dipole, the functions $D_{k l}^{i j}$ are expected to contain a singular term of the second degree. It is important that this is abstracted to permit precise evaluation of the integral equations which will result. This may be done by employing a Taylor series expansion about the source point. The functions $D_{k l}^{i j}$ may then be decomposed into a singular part $D_{k l}^{* i j}$ and a second set of terms $D_{k l}^{\prime i j}$, which are either bounded or more weakly singular:

$$
D_{k l}^{i j}=D_{k l}^{* i j}+D_{k l}^{\prime i j}
$$

It should be noted that terms ${D^{\prime}}_{k l}^{i j}$ are present even in an infinite medium, and for the case of a half-space also include the influence of the free surface. This contrasts with the plane problem, where bounded terms are present only if there are nearby free surfaces. Explicitly, the singular terms are

$$
\begin{aligned}
D_{z z}^{* z z}=\frac{1}{a^{2}}\{ & \frac{2 \cos (2 \psi)-\cos (4 \psi)}{d^{2}} \\
& \left.-\frac{\cos \psi[2 \cos (2 \psi)-\cos (4 \psi)]}{2 d}\right\}
\end{aligned}
$$




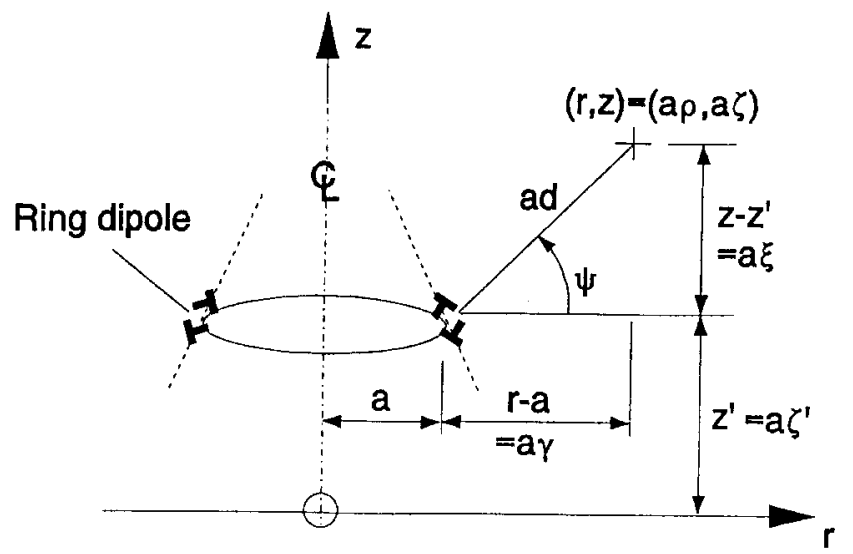

Fig. 3 Notation needed to establish the state of stress near a dipole

$$
\begin{aligned}
& D_{r r}^{* z z}=\frac{1}{a^{2}}\left\{\frac{\cos (4 \psi)}{d^{2}}\right. \\
& \left.-\frac{\cos \psi\left[\cos (4 \psi)+4 \sin ^{2} \psi-2(\kappa-1)\right]}{2 d}\right\} \\
& D_{r z}^{* z z}=\frac{1}{a^{2}}\left[-\frac{2 \sin \psi \cos (3 \psi)}{d^{2}}+\frac{\sin \psi \cos (4 \psi)}{2 d}\right] \\
& D_{z z}^{* r r}=\frac{1}{a^{2}}\left[\frac{\cos (4 \psi)}{d^{2}}-\frac{\cos \psi \cos (4 \psi)}{2 d}\right] \\
& D_{r r}^{* r r}=\frac{1}{a^{2}}\left\{-\frac{2 \cos (2 \psi)+\cos (4 \psi)}{d^{2}}\right. \\
& \left.+\frac{\cos \psi[2 \cos (2 \psi)+\cos (4 \psi)-(\kappa+3)]}{2 d}\right\}
\end{aligned}
$$

$$
\begin{aligned}
D_{r z}^{* r r}=\frac{1}{a^{2}}\{ & -\frac{\sin (2 \psi)+\sin (4 \psi)}{d^{2}} \\
& \left.+\frac{\sin \psi[2 \cos (2 \psi)+\cos (4 \psi)]}{2 d}\right\}
\end{aligned}
$$$$
D_{z z}^{*}=\frac{1}{a^{2}}\left\{\frac{2 \sin \psi \cos (3 \psi)}{d^{2}}\right.
$$$$
\left.-\frac{\sin \psi[2 \cos (2 \psi)+\cos (4 \psi)]}{2 d}\right\}
$$

$$
\begin{aligned}
D_{r r}^{*} r z=\frac{1}{a^{2}}\{ & -\frac{\sin (2 \psi)+\sin (4 \psi)}{d^{2}} \\
& \left.-\frac{\sin \psi[-2 \cos (2 \psi)-\cos (4 \psi)+(\kappa-1)]}{2 d}\right\}
\end{aligned}
$$

$$
D_{r z}^{* r z}=\frac{1}{a^{2}}\left[\frac{\cos (4 \psi)}{d^{2}}-\frac{\cos \psi \cos (4 \psi)}{2 d}\right]
$$

where $\psi$ and $d$ are defined in Fig. 3, and these are normally subtracted from the full expression to facilitate numerical quadrature.

Before proceeding to employ these quantities, some of their characteristics will be listed. The first is that, by making the radius of the dipole very large and the observation point approach the ring, the solution for the corresponding plane dipole may, in each case, be recovered. It may be concluded that the more weakly singular terms present in the ring solutions are present by virtue of the finite curvature of the ring. Secondly, in problems where the crack is inclined at an angle to the centre-line (Fig. 2a), it is necessary to employ a local axis set, oriented parallel to the crack, so that new kernels may be established, relating the traction components of stress to the opening and shear mode dipoles. This may be achieved by noting that both the state of stress and the dipoles themselves transform as second-order tensors, so that the matrix of kernels itself transforms as a fourth-order tensor (Appendix 2).

\section{ANNULAR CRACK}

The simplest problem which may be tackled by this procedure is that of an annular crack, of mean radius $r_{\mathrm{c}}$ and width $2 L$, subjected to arbitrary remote tension and radial shearing stress $\left(\tilde{\sigma}_{z z}(r), \tilde{\sigma}_{r z}(r)\right)$. The problem is shown in Fig. 4, and it is noted, first, that the presence of opening mode ring dipoles induces no shearing tractions in the plane of the ring, as $D_{r z}^{z z}(a ; \rho, 0)=0$ and, similarly, shearing mode dipoles induce no opening mode stress as $D_{z z}^{r z}(a ; \rho, 0)=0$, from equations (8). The problem is therefore reduced to two uncoupled singular integral equations of the form

$$
\begin{gathered}
0=\tilde{\sigma}_{i r}(r)+\frac{2 \mu}{\pi(\kappa+1)} \int_{r_{\mathrm{c}}-L}^{r_{\mathrm{c}}+L} b_{i}(a) D_{i z}^{i z}(a ; \rho, 0) \mathrm{d} a, \\
r_{\mathrm{c}}-L \leqslant a \rho \leqslant r_{\mathrm{c}}+L, i=r, z
\end{gathered}
$$

where the kernels are given by

$$
D_{i z}^{i z}(a ; \rho, 0)=\frac{2 \mathbf{E}}{a^{2}(1+\rho)(1-\rho)^{2}}
$$

where $\mathbf{E}$ is the complete elliptic integral of the second kind given by

$$
\mathbf{E}=\int_{0}^{\pi / 2} \sqrt{1-k^{2} \sin ^{2} \phi} d \phi
$$

with the elliptic modulus 


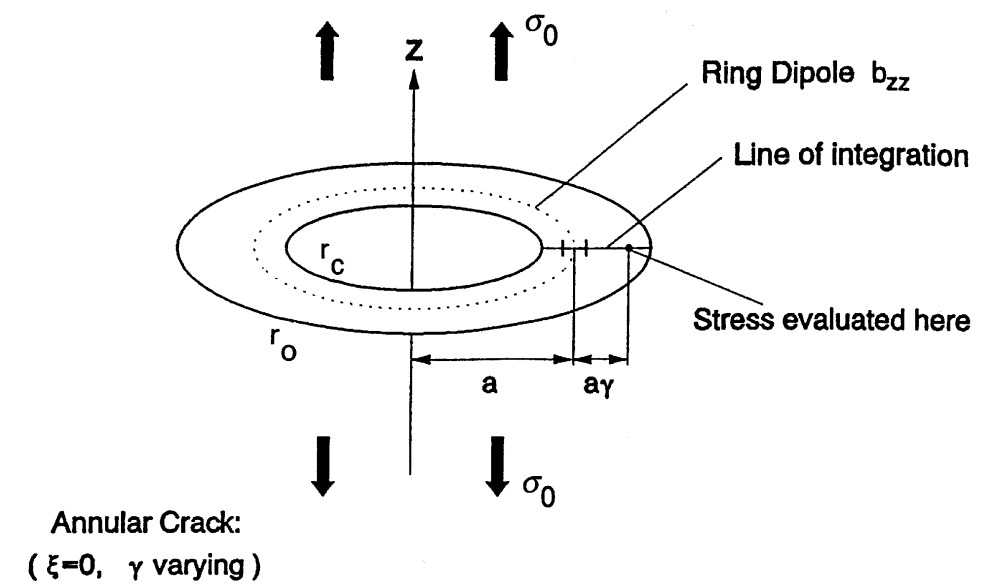

Fig. 4 A flat annular crack in an infinite medium, subject to uniform remote axial tension

$$
k=\frac{2 \sqrt{\rho}}{1+\rho}=\frac{2 \sqrt{a r}}{a+r}
$$

Only the case of a crack subjected to opening mode loading will be treated here, as the shearing case gives rise to very similar equations. The first step in the solution of the above equation is to abstract the singular term, using equations (7), and noting that $\psi=0$, and $d=\rho-1$, so that

$$
\begin{aligned}
D_{z z}^{z z}(a ; \rho, 0)= & {\left[\frac{1}{a^{2}(\rho-1)^{2}}-\frac{1}{2 a^{2}(\rho-1)}\right] } \\
& +D_{z z}^{\prime z z}(a ; \rho, 0)
\end{aligned}
$$

where $D_{z z}^{\prime z z}$ is a bounded function, given by

$$
\begin{aligned}
D_{z z}^{\prime z z}(a ; \rho, 0)= & -\frac{2 \mathbf{E}}{a^{2}(1+\rho)(1-\rho)^{2}} \\
& -\left[\frac{1}{a^{2}(\rho-1)^{2}}-\frac{1}{2 a^{2}(\rho-1)}\right]
\end{aligned}
$$

The integral equation may also be put in standard normalized form by the substitutions

$$
s=\frac{a-r_{\mathrm{c}}}{L}, \quad t=\frac{r-r_{\mathrm{c}}}{L}
$$

so that, with $t-s=(r-a) / L=a(\rho-1) / L$, then

$$
\begin{aligned}
-\frac{\pi L(\kappa+1)}{2 \mu} \tilde{\sigma}_{z z}^{\infty}= & \int_{-1}^{+1} \frac{B_{z z}(s) \mathrm{d} s}{(t-s)^{2}}-\frac{1}{2} \int_{-1}^{+1} \frac{B_{z z}(s) \mathrm{d} s}{\left(s+r_{\mathrm{c}} / L\right)(t-s)} \\
& +\int_{-1}^{+1} B_{z z}(s) D_{z z}^{\prime z z}(t ; s) \mathrm{d} s
\end{aligned}
$$

The regular kernel now reads

JOURNAL OF STRAIN ANALYSIS VOL 35 NO 5

$$
\begin{aligned}
D_{z z}^{\prime z z}(t ; s)= & -\frac{2\left(s+r_{\mathrm{c}} / L\right) \mathbf{E}}{(t-s)\left(s+t+2 r_{\mathrm{c}} / L\right)} \\
& -\left[\frac{1}{(t-s)^{2}}-\frac{1}{2\left(s+r_{\mathrm{c}} / L\right)(t-s)}\right]
\end{aligned}
$$

and the modulus $k$ of the complete elliptic integral $\mathbf{E}$ is given by

$$
k(t ; s)=2 \frac{\sqrt{\left(t+r_{\mathrm{c}} / L\right)\left(s+r_{\mathrm{c}} / L\right)}}{s+t+2 r_{\mathrm{c}} / L}
$$

The singular integral equation (16) contains a hypersingular term of the second degree, a Cauchy term and bounded terms.

The numerical treatment of integral equations with hypersingular kernels of the second degree has been reviewed by Monegato [13] and treated by Kaya and Erdogan [11] and Korsunsky [14]. Without dwelling on details, the basic approaches to the solution of hypersingular equations are mentioned.

The first involves representation of the unknown function by a truncated series of fundamental functions, such as Jacobi polynomials. In many crack problems, because of the behaviour of the unknown function at the ends of the interval, these often reduce to Chebyshev polynomials, which can be treated numerically very effectively, using trigonometric representations. This discretization approach results in a linear algebraic system for the unknown coefficients of the series, with the system matrix populated by quadratures of known functions.

The second method utilizes interpolative Gaussian quadratures. In this formulation, the integrals are expressed as sums over a number of nodal points with certain weights. Both the coordinates of these points and the weights are determined by the behaviour of the unknown function and are once again often related to Chebyshev polynomials [15].

S05199 (C) IMechE 2000 


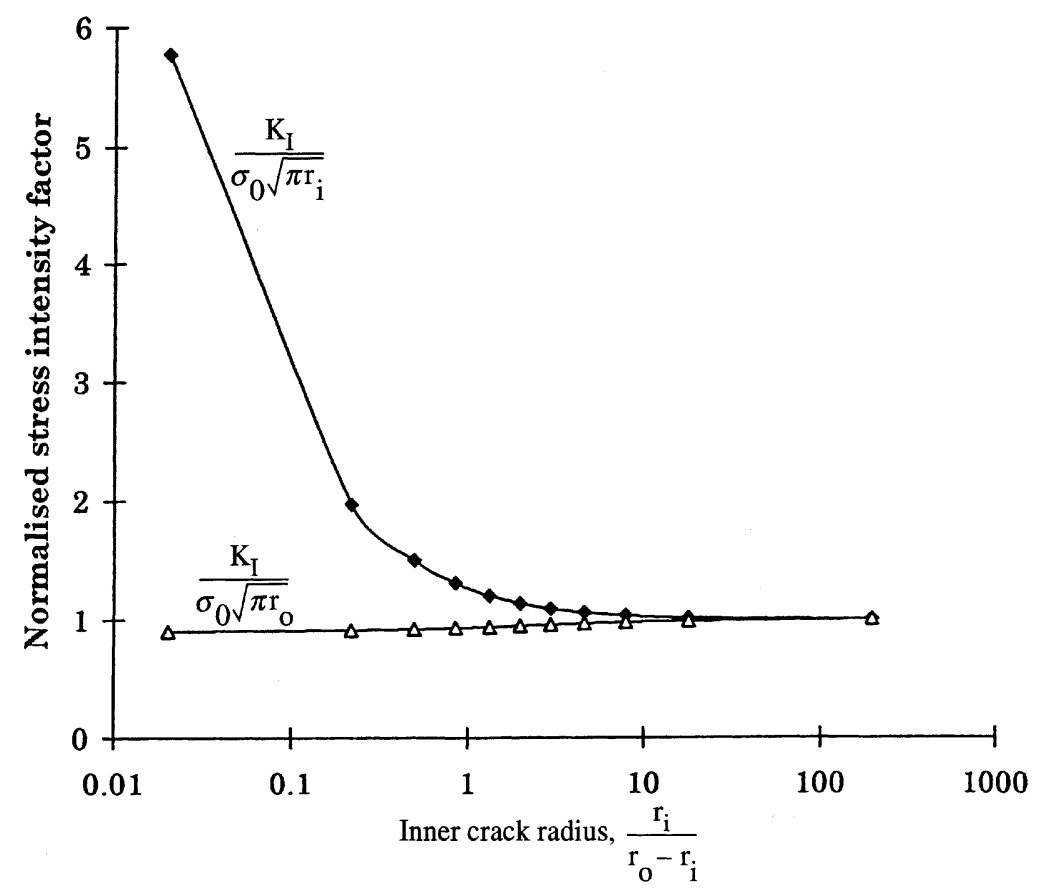

Fig. 5 Stress intensity factors for the crack shown in Fig. 4

The second method usually proves to be more accurate and efficient and is easier to implement, although a greater number of terms is required to achieve given accuracy.

Figure 5 displays results for the stress intensity factor arising at the inner edge of a circular annulus. The results obtained are in good agreement (better than 1 per cent) with the calculations by Clemens and Ang [16].

\section{CYLINDRICAL CRACK}

Now the cylindrical crack shown in Fig. 6 is considered. It is assumed that the crack is subjected to a remote radial tension $\tilde{\sigma}_{z z}(z)$. This will clearly require dipoles $b_{r r}$ to be distributed, but these, in turn, reduce shear stresses $\sigma_{r z}(z)$, and so dipoles of the $b_{r z}$ type will also be required. The integral equations expressing the traction-free requirement in both the radial and axial directions are

$$
\begin{aligned}
&- \frac{\pi(\kappa+1)}{2 \mu} \tilde{\sigma}_{r r}(z) \\
&=\int_{-L}^{+L}\left[B_{r r}\left(z^{\prime}\right) D_{r r}^{r r}(a ; 1, \xi)+B_{r z}\left(z^{\prime}\right) D_{r r}^{r z}(a ; 1, \xi)\right] \mathrm{d} z^{\prime} \\
&-L<z<+L
\end{aligned}
$$

where the kernels are given explicitly in Appendix 1, with $\psi= \pm \pi / 2, \rho=1$ and $d=\left(z-z^{\prime}\right) / a=\xi$. The singular parts of the kernels may be written explicitly from equations (8) and are

$$
\begin{array}{ll}
D_{r r}^{* r r}(a ; 1, \xi)=\frac{1}{a^{2} d^{2}}, & D_{r r}^{* r z}(a ; 1, \xi)=0 \\
D_{r z}^{*}(a ; 1, \xi)=-\frac{1}{a^{2} d^{2}}, & D_{r z}^{* r r}(a ; 1, \xi)=0
\end{array}
$$

It should be noted that the regular part of the kernel in the above expressions may include a weak logarithmic singularity. The stress intensity factors arising at the ends of the crack, subjected to internal pressure, are shown in Fig. 7.

\section{HALF-SPACE PROBLEMS}

It was stated at the outset that the method described above could be employed to solve the problem of axisymmetric cracks in half-spaces, providing that the relevant kernels are employed, which ensure that the surface remains traction-free. These kernels have been found [12,17], but no examples have been included here. The kernels are complex, but contain nothing more complicated than an elliptic integral, or even the slightly more general case of bonded elastically dissimilar half-spaces. 


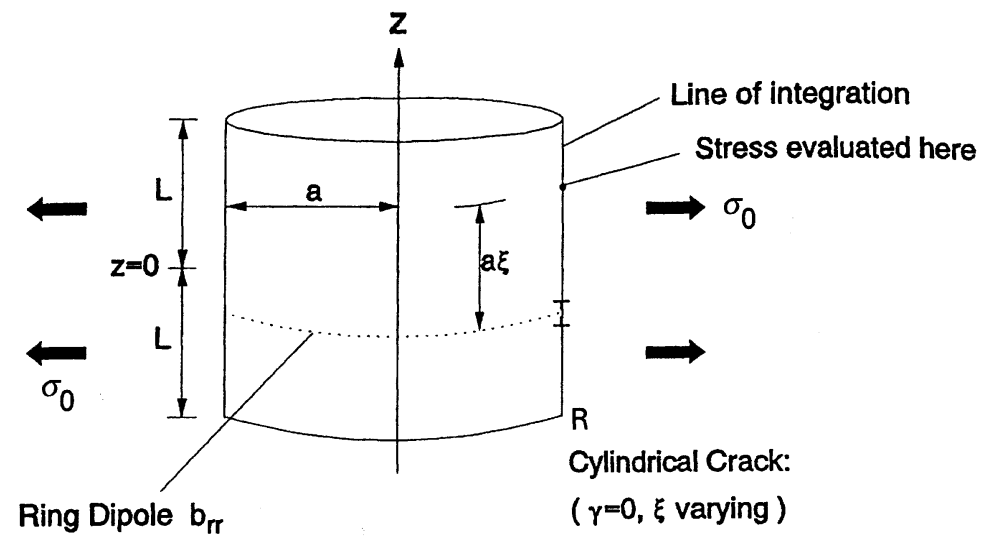

Fig. 6 A cylindrical crack in an infinite medium, subject to uniform remote radial tension

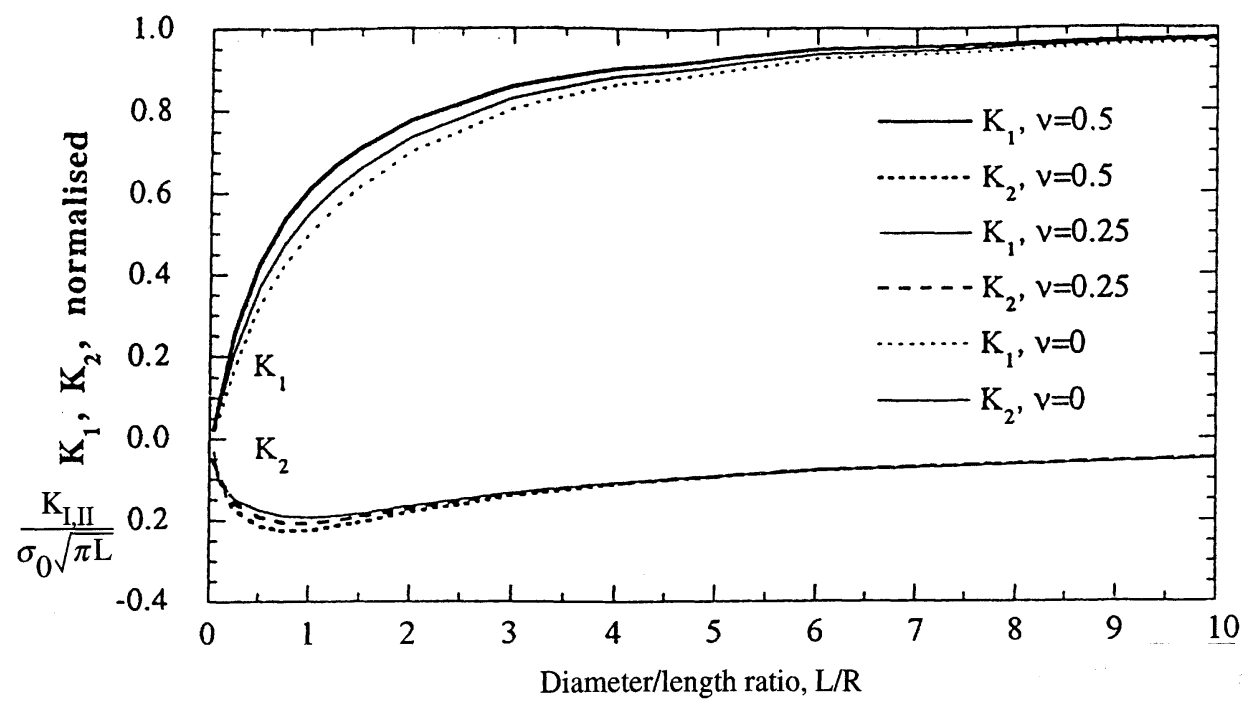

Fig. 7 Stress intensity factors for the crack shown in Fig. 6

\section{DISCUSSION}

The main purpose of this paper is to present a general formulation for axisymmetric crack problems, and to include sufficient detail of the kernel functions involved to enable the reader to construct solutions for a wide range of geometries. Therefore here only a limited set of results for the two problems are presented and introduced as vehicles to illustrate the principles.

The annular crack under remote uniform far-field tension has been treated before [13] and, for all the geometries treated, an accuracy of three significant figures was obtained by a reduction of the integral equation to 20 simultaneous linear algebraic equations, even when the centre-line radius of the crack was only 2 per cent greater than the inner radius, which is the most taxing case. Results were within 1 per cent of those given in reference [13].

Results for the buried cylindrical crack are shown in Fig.
7. These were obtained for several values of Poisson's ratio. It may be noted that the stress intensity factors found depend on this elastic constant, in contrast with the corresponding plane problem.

\section{CONCLUSION}

A method for the solution of cracks of axisymmetric shape present in simple geometries, such as an infinite space or bonded half-spaces, has been described. It is based on an integral equations formulation, using pairs of Somigliana ring dislocations, arranged so as to form dipoles, as the kernel. This process leads to the formulation of tractable integral equations, whose dominant term is hypersingular to the second degree. These may be reduced by recently developed methods to families of linear algebraic equations, whose ready inversion means that solution of the underlying 
problem is computationally very efficient. This is particularly so in the case of the half-space types of problem, as boundary conditions on the free surface are incorporated into the kernels and are therefore automatically satisfied.

\section{REFERENCES}

1 Bueckner, H. F. The propagation of cracks and the energy of elastic deformation. J. Appl. Mechanics, 1958, 80, 12251230 .

2 Erdogan, F. and Gupta, G. D. On the numerical solution of singular integral equations. Q. Appl. Math., 1972, 30, 525534.

3 Erdogan, F., Gupta, G. D. and Cooke, T. S. Numerical solution of singular integral equations. In Methods of Analysis and Solution of Crack Problems (Ed. G. C. Sih), 1973, pp. 368-425 (Noordhoff, Leyden).

4 Nowell, D. and Hills, D. A. Open cracks at or near free edges. J. Strain Analysis, 1987, 22(3), 177-185.

5 Krenk, S. On quadrature formulae for singular integral equations of the first and second kind. Q. Appl. Math., 1975, 33, 225-232.

6 Korsunsky, A. M. and Hills, D. A. The solution of crack problems by using distributed strain nuclei. Proc. Instn Mech. Engrs, Part C, Journal of Mechanical Engineering Science, 1995, 210(C1), 23-31.

7 Crouch, S. L. Solution of plane elasticity problems by the displacement discontinuity method. Int. J. Numer. Meth. Engng, 1976, 10, 301-343.

8 Zhao, M. H., Liu, Y. J. and Cheng, C. J. Boundary integral equations and the boundary element method for threedimensional fracture mechanics. Engng Analysis Boundary Elements, 1994, 13, 333-338.

9 Guiggiani, M., Krishnasamy, G., Rudolphi, T. J. and Rizzo, F. I. A general algorithm for the numerical solution of hypersingular boundary integral equations. J. Appl. Mechanics, 1992, 59, 604-614.

10 Ioakimidis, N. I. Application of finite-part integrals to the singular integral equations of crack problems in plane and three-dimensional elasticity. Acta Mechanica, 1982, 45, 3147.

11 Kaya, A. C. and Erdogan, F. On the solution of singular integral equations with strongly singular kernels. Q. Appl. Math., 1987, 45, 105-122.

12 Korsunsky, A. M. Fundamental eigenstrain solutions for axisymmetric crack problems. J. Mechanics Physics Solids, 1995, 43, 1221-1241.

13 Monegato, G. Numerical evaluation of hypersingular integrals. J. Comput. Appl. Math., 1994, 50, 9-31.

14 Korsunsky, A. M. The solution of axi-symmetric crack problems in inhomogeneous media. DPhil thesis, University of Oxford, 1994.

15 Korsunsky, A. M. Gauss-Jacobi quadratures for hypersingular integrals. In Proceedings of the First UK Conference on Boundary Integral Methods (Eds L. Elliott, D. B. Ingham and D. Lesnic), 1997, pp. 164-177 (Leeds University Press, Leeds).

16 Clemens, D. L. and Ang, W. T. Stress intensity factors for the circular annular crack. Int. J. Engng Sci., 1988, 26, 325-329.
17 Murakami, Y. Stress Intensity Factors Handbook, Vol. 3, 1993 (Pergamon, Oxford).

\section{APPENDIX 1}

\section{Influence functions for ring dipoles in an infinite medium}

The influence functions $D_{k l}^{i j}$ below give the stress components $\sigma_{k l}$ at point $r, z$ due to the ring dipoles $b_{i j}$ of unit intensity. The ring dipoles of radius $a$ are centred at $r=0$, $z=z^{\prime}$, and lie in an infinite elastic medium with the modulus of rigidity $\mu$ and Kolosov's constant $\kappa=(3-4) v$, where $v$ is Poisson's ratio. The dimensionless coordinates are introduced by

$$
\begin{aligned}
\rho=\frac{r}{a}, \quad \xi & =\frac{z-z^{\prime}}{a} \\
D_{z z}^{z z}(a ; \rho, \xi)= & \frac{\pi}{a^{2}}\left[-J_{002}(\rho, \xi)-\xi J_{003}(\rho, \xi)\right] \\
D_{r r}^{z z}(a ; \rho, \xi)=\frac{\pi}{a^{2}} & {\left[-J_{002}(\rho, \xi)+\xi J_{003}(\rho, \xi)-\frac{\xi}{\rho} J_{012}(\rho, \xi)\right.} \\
& \left.+\frac{\kappa-1}{2 \rho} J_{011}(\rho, \xi)\right]
\end{aligned}
$$$$
D_{r z}^{z z}(a ; \rho, \xi)=\frac{\pi}{a^{2}}\left[-\xi J_{013}(\rho, \xi)\right]
$$$$
D_{z z}^{r r}(a ; \rho, \xi)=\frac{\pi}{a^{2}}\left[-J_{002}(\rho, \xi)+\xi J_{003}(\rho, \xi)\right]
$$$$
D_{r r}^{r r}(a ; \rho, \xi)=\frac{\pi}{a^{2}}\left[3 J_{002}(\rho, \xi)-\xi J_{003}(\rho, \xi)\right.
$$$$
\left.+\frac{\xi}{\rho} J_{012}(\rho, \xi)-\frac{3+\kappa}{2 \rho} J_{011}(\rho, \xi)\right]
$$$$
D_{r z}^{r r}(a ; \rho, \xi)=\frac{\pi}{a^{2}}\left[-2 J_{012}(\rho, \xi)+\xi J_{013}(\rho, \xi)\right]
$$$$
D_{z z}^{r z}(a ; \rho, \xi)=\frac{\pi}{a^{2}}\left[-\xi J_{103}(\rho, \xi)\right]
$$$$
D_{r r}^{r z}(a ; \rho, \xi)=\frac{\pi}{a^{2}}\left[-2 J_{102}(\rho, \xi)+\xi J_{103}(\rho, \xi)\right.
$$$$
\left.-\frac{\xi}{\rho} J_{112}(\rho, \xi)+\frac{\kappa+1}{2 \rho} J_{111}(\rho, \xi)\right]
$$$$
D_{r z}^{r z}(a ; \rho, \xi)=\frac{\pi}{a^{2}}\left[J_{112}(\rho, \xi)-\xi J_{113}(\rho, \xi)\right]
$$

The quantities $J_{m n p}$ are the Lipschitz-Hankel integrals. 


\section{APPENDIX 2}

\section{Influence function transformation rules}

Introduce a local system of coordinates associated with the ring, which is rotated an angle $\theta$ counterclockwise with respect to the global system $(r, z)$, i.e. define

$$
\begin{aligned}
& x=r \cos \theta+\left(z-z^{\prime}\right) \sin \theta \\
& y=-r \sin \theta+\left(z-z^{\prime}\right) \cos \theta
\end{aligned}
$$

Consider the family of ring dipoles of geometry defined in the previous section. Let the dipoles be combined so as to produce displacement discontinuity components in the local frame associated with elemental surfaces defined by unit normals along the $x$ and $y$ axes. In order to evaluate the stresses generated by these fundamental ring dipoles in the local coordinate system, tensor transformation rules must be applied.

The influence functions $\hat{D}_{p q}^{m n}$ due to a dipole ring $\hat{\mathrm{b}}_{m n}$ of unit intensity, which is defined with respect to the local coordinate system, give the expressions for the stresses $\hat{\sigma}_{p q}$ in this system. These influence functions are calculated using

$$
\hat{D}_{p q}^{m n}=D_{k l}^{i j} a_{i m} a_{j n} a_{k p} a_{l q}
$$

where the summation convention applies for repeated indices, and $a_{i m}$ are the components of the rotation tensor

$$
\mathbf{A}=\left\{\begin{array}{cc}
\cos \theta & \sin \theta \\
-\sin \theta & \cos \theta
\end{array}\right\}
$$

